\title{
A comparison of strength for two continuous peripheral nerve block catheter dressings
}

\author{
Lindsay Borg $^{1}$, Steven K. Howard ${ }^{1,2}$, T. Edward Kim ${ }^{1,2}$, Lauren Steffel ${ }^{1}$, \\ Cynthia Shum ${ }^{2}$, and Edward R. Mariano ${ }^{1,2}$ \\ ${ }^{I}$ Department of Anesthesiology, Perioperative and Pain Medicine, Stanford University School of Medicine, Stanford, \\ ${ }^{2}$ Anesthesiology and Perioperative Care Service, Veterans Affairs Palo Alto Health Care System, Palo Alto, CA, USA
}

Background: Despite the benefits of continuous peripheral nerve blocks, catheter dislodgment remains a major problem, especially in the ambulatory setting. However, catheter dressing techniques to prevent such dislodgment have not been studied rigorously. We designed this simulation study to test the strength of two commercially available catheter dressings. Methods: Using a cadaver model, we randomly assigned 20 trials to one of two dressing techniques applied to the lateral thigh: 1) clear adhesive dressing alone, or 2) clear adhesive dressing with an anchoring device. Using a digital luggage scale attached to a loop secured by the dressing, the same investigator applied steadily increasing force with a downward trajectory towards the floor until the dressing was removed or otherwise disrupted.

Results: The weight, measured (median [10th-90th percentile]) at the time of dressing disruption or removal, was 1.5 $\mathrm{kg}(1.3-1.8 \mathrm{~kg})$ with no anchoring device versus $4.9 \mathrm{~kg}(3.7-6.5 \mathrm{~kg})$ when the dressing included an anchoring device $(\mathrm{P}<$ $0.001)$.

Conclusions: Based on this simulation study, using an anchoring device may help prevent perineural catheter dislodgement and therefore premature disruption of continuous nerve block analgesia.

Key Words: Anchoring device, Continuous peripheral nerve block, Dislodgement, Dressing, Perineural catheter, Regional anesthesia.

\section{Introduction}

Despite the benefits of continuous peripheral nerve blocks (CPNB), catheter dislodgment remains a major problem, es-

Corresponding author: Edward R. Mariano, M.D., M.A.S.

Anesthesiology and Perioperative Care Service, Veterans Affairs Palo Alto Health Care System, 3801 Miranda Avenue (112A), Palo Alto, CA 94304, USA

Tel: 1-650-849-0254, Fax: 1-650-852-3423

E-mail: emariano@stanford.edu

ORCID: http://orcid.org/0000-0003-2735-248X

Received: April 26, 2016.

Revised: May 28, 2016.

Accepted: May 31, 2016.

Korean J Anesthesiol 2016 October 69(5): 506-509

http://dx.doi.org/10.4097/kjae.2016.69.5.506 pecially in the ambulatory setting. Catheter dislodgement, and therefore interruption of analgesia, is one of the most common undesirable outcomes of CPNB, with reported dislodgement rates of up to $30 \%$ [1]. In a recent retrospective comparison of failure rates among upper extremity blocks, Ahsan et al. [2] reported CPNB failure rates as high as $26 \%$ for supraclavicular, and $19 \%$ for infraclavicular, brachial plexus catheters. They proposed several potential reasons for block failure, including genetic variation in local anesthesia metabolism and improper initial catheter placement, but concluded that catheter dislodgement away from the target nerve was a likely cause for block failure in their study [2]. They noted a higher failure rate in perineural catheters placed above versus below the clavicle, and attributed this to differences in catheter securement and muscle availability at the site of insertion (i.e., to anchor the catheter and thus prevent dislodgement) [2]. Another study of healthy

(c) This is an open-access article distributed under the terms of the Creative Commons Attribution Non-Commercial License (http://creativecommons.org/ licenses/by-nc/4.0/), which permits unrestricted non-commercial use, distribution, and reproduction in any medium, provided the original work is properly cited. 
volunteers reported catheter tip dislodgment rates of 5\% for interscalene catheters and $25 \%$ for femoral nerve catheters after a period of standardized physical activity [3].

While many perineural catheter dressing and securement strategies have been described, including tunneling, topical skin adhesive, sterile tape, clear adhesive dressings, and anchoring devices [1,4-6], rigorous head-to-head comparisons of these strategies have not been reported. Thus, the optimal perineural catheter dressing strategy to ensure reliable infusion of local anesthetic for the desired duration remains unknown. We designed this cadaver-based study to objectively compare the strength of two commonly used perineural catheter dressing and securement methods.

\section{Materials and Methods}

After obtaining Institutional Review Board exemption from review and Veterans Affairs research committee approval, we conducted this pilot study using one unembalmed male cadaver weighing $44 \mathrm{~kg}$ and measuring $160 \mathrm{~cm}$ in height. Twenty catheter securement trials were randomly assigned (www.randomizer. org) to one of two groups based on dressing technique: 1) clear adhesive dressing alone (Tegaderm; 3M, St. Paul, MN, USA), or 2) clear adhesive dressing with an anchoring device (Statlock; Bard Medical, Covington, GA, USA).

For each trial, the same epidural catheter connector (SnapLock; Teleflex Medical, Research Triangle Park, NC, USA) attached to Luer-lock plastic tubing fashioned in the form of a loop (Fig. 1) was secured and dressed, according to the randomization assignment, on the lateral aspect of the cadaver's right

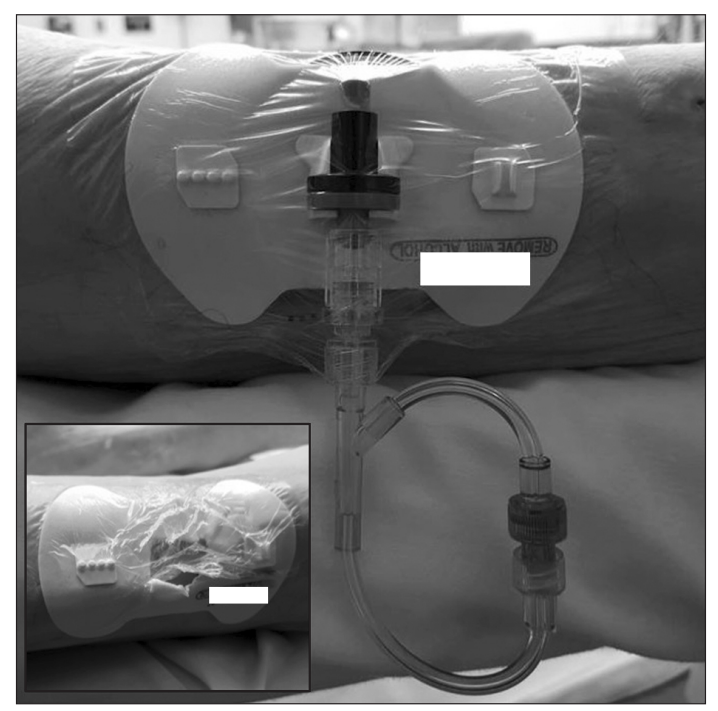

Fig. 1. Image showing the experimental model used to test the clear adhesive dressing with an anchoring device. Inset box: image showing the simulated dressing after disruption caused by application of force. thigh at a site between the greater trochanter of the femur and the top of the patella. We did not use an actual catheter for the final experiment: in pilot trials, we attempted to use a catheter but found it to be too elastic to permit measurement of weight. Thus, we created a loop of Luer-lock plastic tubing that could be attached to the catheter connector (Fig. 1). This catheter connector accommodates the end of the catheter and snaps into the anchoring device (Statlock). The anchoring device has adhesive backing that is affixed to the skin. In the trials randomized to include an anchoring device, a large clear adhesive dressing (Tagaderm) was applied on top of the anchoring device as a cover (Fig. 1). In the trials randomized to clear adhesive dressing (Tagaderm) alone, the catheter connector was affixed to the skin directly without the aid of an anchoring device.

Using a digital luggage scale equipped with a hook (Naftali Inc., Miami Gardens, FL, USA), the same investigator applied steadily increasing force to the tubing loop with a downward trajectory towards the floor, until the dressing was removed or otherwise disrupted, resulting in an unsecured catheter connector (Fig. 1, inset box). Although the same right lateral thigh was used for all trials, the actual site of adhesion differed from trial to trial. A new clear adhesive dressing (Tagaderm) and new anchoring device (Statlock) were used in each trial when applicable.

The primary endpoint was the weight measured $(\mathrm{kg})$ by the scale at the time of dressing disruption or removal. Normality of distribution was determined using the Kolmogorov-Smirnov test. The outcome data were not normally distributed; thus, they were compared using the Mann-Whitney $U$ test. $P$ values $<0.05$ were considered to indicate statistical significance.

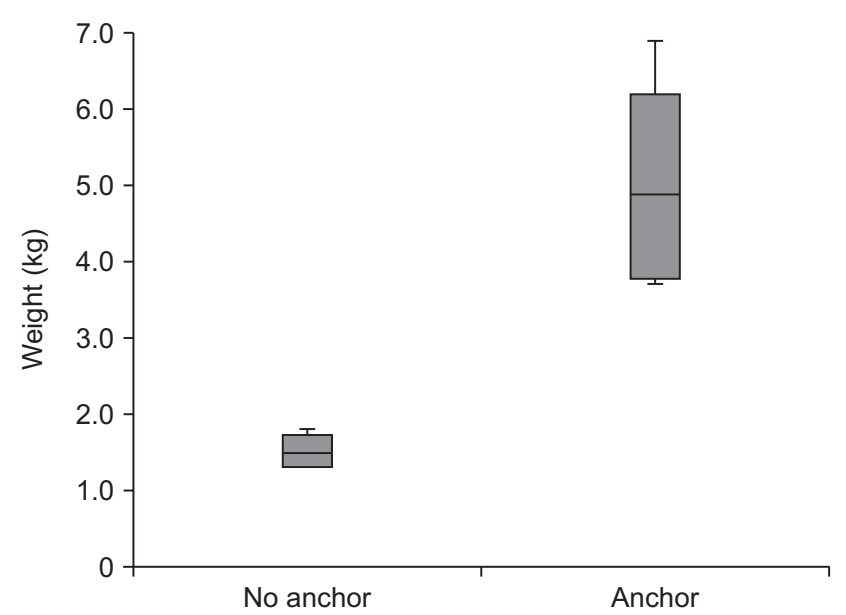

Fig. 2. Weight measured $(\mathrm{kg})$ at the time of dressing disruption or removal. Boxes indicate the 10th-90th percentiles; whiskers indicate the range. 


\section{Results}

All 20 dressing trials were completed successfully. The skin of the cadaver remained intact throughout the study. The weight measured (median [10th-90th percentiles]) at the time of dressing disruption or removal was 1.5 (1.3-1.8) $\mathrm{kg}$ for the clear dressing alone (i.e., with no anchoring device) versus 4.9 (3.7-6.5) $\mathrm{kg}$ when the dressing included an anchoring device $(\mathrm{P}<0.001$; Fig. 2).

\section{Discussion}

The results of this pilot study clearly show that a perineural catheter dressing technique using an anchoring device with a clear adhesive cover withstood over three times the weight of a clear adhesive dressing alone. Based on our simulations, using an anchoring device may help prevent perineural catheter dislodgement and by extension premature disruption of continuous nerve block analgesia.

Previous studies have shown perineural catheter dislocation and dislodgement to be a clinically relevant problem, especially for ambulatory surgery patients [2]. The duration of planned CPNB treatment may correlate with an increasing risk of catheter dislodgment [3]. One in four femoral nerve block catheter dislocations occurred within $6 \mathrm{~h}$ of the initial placement [3], suggesting that the actual dislocation rate for patients who go home with perineural infusions intended to last 2-3 days in clinical practice may be much higher [7].

There are several limitations to this study. First, we employed a simulation model to test dressing strength, rather than using actual clinical patients or live human subject volunteers. Although the cadaver was unembalmed, the elastic properties of the cadaver skin and its interaction with our two dressings clearly differed to the skin of a live patient. However, these study procedures may have caused severe discomfort in a live human subject. Additionally, the study tested dressing strength by using a weight sufficient to disrupt the integrity of the dressing but not cause actual catheter dislocation or dislodgement. Previous studies have investigated catheter migration and dislocation in a simulation setting by using cadavers and volunteers $[3,8,9]$, but the present study is the first to rigorously compare perineural catheter dressing and securement methods.

We agree with Ahsan et al. [2] that the causes of secondary block failure during planned CPNB treatment are likely multifactorial, but the method of catheter securement may be an important factor not previously tested. Any disruption of the integ- rity of the external dressing by force applied to the distal end of the catheter may result in movement of the catheter at the point of insertion, or internally. In out study, only the cadaver's lateral thigh was used, approximating the site where a popliteal-sciatic perineural catheter would likely be anchored, and no other site was tested; therefore, the results of this study cannot be applied generally to other perineural catheter locations. Additionally, this simulation model relied on testing only one mechanism of catheter dressing disruption or removal. In reality, catheters may be accidentally dislodged through other methods that may involve various levels of force and velocity (e.g., attaching the pump to the bed and walking away). The simulated catheter was simply pulled upon by an investigator. More complex and realistic mechanisms of catheter dressing disruption, which could have been achieved by manipulating the simulated range of motion or patient activity, were not attempted. Whether a threefold difference in dressing strength corresponds to a clinically relevant difference in CPNB catheter dislodgement rates is also unknown.

Finally, we only tested two dressing and securement strategies, both involving clear adhesive dressing; thus, the results of this study are specific to the techniques and materials tested. We do not speculate on how other methods, such as tunneling, and application of liquid adhesives or surgical tape, among others $[5,6]$, may have performed either alone or in combination. Previous publications have asserted that a combination of several methods may lead to successful perineural catheter retention for a week or longer [4,10-12].

In summary, catheter dislodgement remains an important and frequent cause of CPNB failure, leading to premature disruption of postoperative regional analgesia. Dressing technique plays an important role in the prevention of catheter dislodgment but has not been formally studied prior to the present investigation, which suggests that use of an anchoring device may strengthen dressing integrity and therefore reduce the potential for catheter dislodgement. Further studies investigating other common catheter securing strategies, different anatomical sites, and possibly using live patients, will be helpful in determining the optimal dressing for CPNB.

\section{Acknowledgments}

The authors gratefully acknowledge the assistance of Mr. Russell Sanchez and the Pathology Service, Veterans Affairs Palo Alto Health Care System (Palo Alto, CA, USA). 


\section{References}

1. Ilfeld BM. Continuous peripheral nerve blocks in the hospital and at home. Anesthesiol Clin 2011; 29: 193-211.

2. Ahsan ZS, Carvalho B, Yao J. Incidence of failure of continuous peripheral nerve catheters for postoperative analgesia in upper extremity surgery. J Hand Surg Am 2014; 39: 324-9.

3. Marhofer D, Marhofer P, Triffterer L, Leonhardt M, Weber M, Zeitlinger M. Dislocation rates of perineural catheters: a volunteer study. Br J Anaesth 2013; 111: 800-6.

4. Ilfeld BM. Continuous peripheral nerve blocks: a review of the published evidence. Anesth Analg 2011; 113: 904-25.

5. Boezaart AP. Continuous interscalene block for ambulatory shoulder surgery. Best Pract Res Clin Anaesthesiol 2002; 16: 295-310.

6. de José María B, Banús E, Navarro-Egea M, Banchs RJ. Tips and tricks to facilitate ultrasound-guided placement of peripheral nerve catheters in children. Paediatr Anaesth 2011;21: 974-9.

7. Levine M, Latmore M, Vandepitte C, Gadsden J, Hadzic A. It is 3 a.m....do you know where your catheter tip is? Br J Anaesth 2014; 112: 757-8.

8. Webb CA, Kim TE, Funck N, Howard SK, Harrison TK, Ganaway T, et al. Comparison of catheter tip migration using flexible and stimulating catheters inserted into the adductor canal in a cadaver model. J Anesth 2015; 29: 471-4.

9. Leng JC, Harrison TK, Miller B, Howard SK, Conroy M, Udani A, et al. A pilot study to assess adductor canal catheter tip migration in a cadaver model. J Anesth 2015; 29: 308-12.

10. Ang ET, Lassale B, Goldfarb G. Continuous axillary brachial plexus block--a clinical and anatomical study. Anesth Analg 1984; 63: 680-4.

11. Ilfeld BM, Wright TW, Enneking FK, Mace JA, Shuster JJ, Spadoni EH, et al. Total shoulder arthroplasty as an outpatient procedure using ambulatory perineural local anesthetic infusion: a pilot feasibility study. Anesth Analg 2005; 101: 1319-22.

12. Stojadinovic A, Auton A, Peoples GE, McKnight GM, Shields C, Croll SM, et al. Responding to challenges in modern combat casualty care: innovative use of advanced regional anesthesia. Pain Med 2006; 7: 330-8. 